076.

\section{Lesson from the Hokkaido Southwestern Offshore Earthquake}

Asai Yasufumi, Kaneko Masamitsu, Nara Satoshi, Mori Kazuhisa, Sakano Shoji, Yoshida Masashi, Ito Yasushi, Naito Takafumi, Imaizumi Hitoshi, Kobayashi Kenji, Shöi Tetsuro

Department of Traumatology and Critical Care Medicine, Sapporo Medical University School of Medicine,

Sapporo, Japan

Introduction: On 12 July 1993 at 22:17 hours, a magnitude 7.8 earthquake and a subsequent tsunami struck a wide area of the northern part of Japan. The heaviest damage occurred on the small island of Okushiri, located in the epicenter area, 30 $\mathrm{km}$ west of the island of Hokkaido. The majority of the casualties and damage were due to the tsunami. The problem of medical support, life lines, and the role of mass communication are discussed.

Results: As of June 1994, 229 deaths were reported. Twentyeight people were killed by landslides; the rest were killed by drowning. A total of 83 persons were severely injured, and 240 sustained mild injures. In the Okushiri district, four doctors in a local hospital provided treatment for 25 severely injured and 44 lightly injured patients. However, no doctors were present in the Aonae District. Medical and rescue teams of the Ground Self-Defense Force from Sapporo took eight hours to arrive at Aonae because of bad weather. We treated seven patients who wee transferred by helicopter. The majority of cases sustained bone fractures. They also needed counseling for emotionally induced "mental" scars. In this disaster, mass communications played an important role to announce these patients' present condition and necessity for rescue.

Conclusions: 1) Confusion on the appropriate use of the communication network such as telephone and wireless was extreme. Therefore, the exclusive use of the wireless is necessary for medical communications. 2) Dispatch of medical teams to those areas without doctors by helicopter is needed as soon as possible to perform triage, treatment, and transportation. 3) There is a need to establish a national organization for disaster such as the FEMA (Federal Emergency Management Agency) in the USA. 4) The Ground Self-Defense Force should play an important role in disaster management.

\section{6.}

\section{The Tasks of the Swedish Rescue Services Agency}

\section{Monica Andersson}

Swedish Rescue Services Agency, Karlstad, Sweden

The Swedish Rescue Services Agency is the central authority which, together with the municipal rescue service, is responsible for population protection and rescue services in society. Within this area of responsibility, the agency promotes work to prevent accidents and reduce damage. In this way, the agency contributes to make our society safer.

The Swedish Rescue Service Agency participates not only in national safety work, but also on the international scene. The need for humanitarian efforts is expected to rise. By maintaining a high level of preparedness for aid in case of disasters internationally, the agency supports the people and those responsible for rescue service in other countries. This is done, for example, by supporting the Baltic States. To contribute to developing rescue service and safety work in other countries, the agency has an increasing export of services. To be able to perform these activities, the Swedish Rescue Service Agency has divided its operation into five branches: 1) public rescue services; 2) safety of operations involving special risks; 3) capability of the individual; 4) population protection in times of war; and 5) international activities.

The agency's preparedness in aid/disaster relief within the framework of UNHCR is organized into four fields according to aid required: 1) "Rescue Team" for rescue operations; 2) "Support Team" for relief operations at acute refugee-producing disasters; 3) "Emergency Team" for supply operations; and 4) "Expert Team" for expert operations.

\section{0.}

\section{The Prehospital Rescue Organization: A New Way to Integrate Resources}

\author{
Maurizo Barbero, MD, Francesco Bermano, Salvatore Esposito, \\ Fiorella Robba \\ Emergency Center, S. Paul Hospital, Savona, Italia
}

A prehospital emergency always has represented a crucial point in the health-care system. Human and technical resources should be used rationally to improve the efficiency of a prehospital rescue system. An efficient system must be able to provide equipment, means of transport, and an experienced staff at the scene of the accident as soon as possible.

The Savona Emergency Department has had a modern assistance vehicle (rescue car) for several years. It's a small, but easy-to-handle Fiat Panda with four-wheel drive that is well equipped in anticipation of a necessary prehospital resuscitation. The rescue car is dispatched from the hospital and guarantees a fast intervention only within our territorial area.

There is considerable summer tourism in our region (also present in 1994) that required a potentate prehospital emergency service. This is why we had to set up decentralized firstaid centers. Because of the number of tourists and the problem of distances, three peripheral centers have been established. Every center was provided with a rescue car, a specially trained physician, and a specially trained nurse who was called during an emergency. The physicians working in the above-mentioned centers staffed the first-aid stations and the nurses came from the emergency staff or were part of other departments. The activities of these peripheral centers from the 01 July to 31 August 1994 are listed in the following table:
Global Analysis
Total services
2,772
Ambulatory services
2,657
(53\% internal medicine and $47 \%$ surgical)
Rescue-car service 